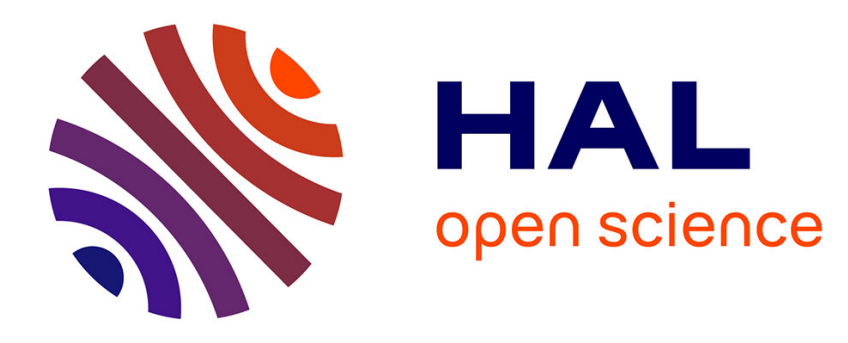

\title{
GIANT RESONANCES IN HEAVY ION COLLISIONS
}

$\mathrm{Ph}$. Chomaz

\section{To cite this version:}

Ph. Chomaz. GIANT RESONANCES IN HEAVY ION COLLISIONS. International Conference on Heavy Ion Nuclear Collisions in the Fermi Energy Domain Hicofed 86, 1986, Caen, France. pp.C4155-C4-170, 10.1051/jphyscol:1986418 . jpa-00225784

\section{HAL Id: jpa-00225784 https://hal.science/jpa-00225784}

Submitted on 1 Jan 1986

HAL is a multi-disciplinary open access archive for the deposit and dissemination of scientific research documents, whether they are published or not. The documents may come from teaching and research institutions in France or abroad, or from public or private research centers.
L'archive ouverte pluridisciplinaire HAL, est destinée au dépôt et à la diffusion de documents scientifiques de niveau recherche, publiés ou non, émanant des établissements d'enseignement et de recherche français ou étrangers, des laboratoires publics ou privés. 


\title{
GIANT RESONANCES IN HEAVY ION COLLISIONS
}

\author{
Ph. CHOMAZ \\ Division de Physique Théorique*, Institut de Physique \\ Nucléaire, F-91406 Orsay Cedex, France
}

Résumé - Dans cet article nous discutons les progrès apportés par les ions Tourds dans la connaissance des résonances gêantes. Cette discussion s'appuie sur 1a description de trois résultats récents et sur la comparaison de ces résultats avec les prédictions des modèles microscopiques (RPA, quasi-boson et multiphonon ....). Nous montrons comment les mesures de décroissance par raies $\gamma$ sont un test de la structure des résonances geaantes et nous décrivons des expériences qui ont mis en évidence soit des résonances de très haute énergie soit des états de multiphonons bâtis sur les résonances géantes à 2 hw d'énergie d'excitation. Nous abordons aussi le rôle des rêsonances géantes dans les réactions par ions lourds et à la lumière des nouveaux résultats présentês nous mettons 1 'accent sur 1 'importance de l'excitation des multiphonons dans les mécanismes de réactions par ions lourds.

Abstract - This paper presents some of the new results on giant resonances obtained using heavy ion projectiles. It is shown that the microscopic structure of giant states can be probed using $\gamma$ coincidence measurements. An example of the search for high lying states with light "heavy. ion" probes at high incident energy is given and, the investigation of multiphonon excitation using heavier ions at intermediate energy is discussed. These experimental results are compared with the theoretical predictions of the multiphonon mode1. The role of giant resonances in heavy ion reactions is discussed and in the light of the new experimental results the importance of the multiphonon excitation for the heavy ion dynamics is emphasized.

\section{I - INTRODUCTION}

Since the discovery of the giant dipole resonance GDR in photoabsorption reactions in $1947 / 1 /$ a considerable amount of experimental and theoretical efforts have been devoted to the study of giant resonances $/ 2 \%$. In particular, during the last decade numerous vibrational modes have been observed and studied by means of inelastic scattering of electrons and hadrons ( $\left.p, d, 3_{\mathrm{He},}^{4} \mathrm{He}\right)$. These modes are seen to be a general property of nuclei, they are coupled to the ground state by strong electro-magnetic matrix elements, their excitation energies and widths vary smoothly with the nuclear mass $A$ ( $i$.e. 1 ike $A^{-1 / 3}$ and $A^{-2 / 3}$ respectively). Giant resonances are expected to play a dominant role in many nuclear reactions. For example the excitation of collective states in heavy ion reactions is now well established $/ 3-5 /$. The purpose of this paper is to study, on the one hand, the role of giant resonances in heavy ion collision mechanisms and, on the other hand, the way in which heavy ion collisions can enhance our knowledge of giant resonance states. A complete review of these two points is nearly an impossible task and thus I shall restrict myself to the discussion of a few selected aspects. After a rapid introduction on the nature of giant resonances I sha 11 touch in section II upon the role of giant resonances in heavy ion collisions. Then in section III I shall

\footnotetext{
* Laboratoire associé au CNRS
} 
discuss recent or future studies on giant resonances performed with heavy ion probes: subsection III.1 will be devoted to the study of giant state decay whereas subsection III.2 will describe the observation of new giant states at high excitation energy. The implications of this new information on the role of giant states in heavy ion dynamics will then be stressed and conclusions wi11 be drawn in the last section.

\section{II - ROLE OF GIANT RESONANCES}

\section{II.1. - Generalities.}

In the liquid-drop model giant resonances are interpreted as collective vibrations of the nucleus. For example, the monopole resonance is a compression mode whereas the other modes are associated with nuclear surface deformations $/ 6 /$. In electric isoscalar vibrations all nucleons vibrate in phase, conversely in isovector modes protons and neutrons vibrate out of phase as do spin-up and spindown nucleons in magnetic resonances.

In a microscopic description, giant resonances can be seen as coherent excitations of elementary particle-hole transitions in a she11 mode1. Hence the properties of the resonance : the multipolarity, the strength, the spin and isospin transfers are defined by the underlying particle-hole structure. This structure is often described by the Random Phase Approximation (R.P.A).

In fact the two previous descriptions of giant states are complementary and it is often useful to consider both in order to get a better understanding of giant resonance properties.

\section{II.2. - Zero point motions.}

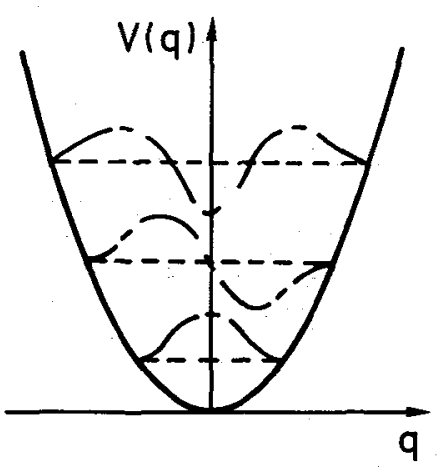

Fig. 1
Giant resonances induce zero point motion in the collective coordinate $q$. Indeed, a giant resonance can be considered as a quantum harmonic oscillator in the coordinate $q$. Fiaure 1 presents the three first states in a parabolic potential together with their wave function. The extention in $q$ of the wave function of the first state illustrates the zero point motion of the nucleus in its ground state. The two other states are respectively the one-phonon (i.e. the giant resonance itse $7 f$ ) and the two phonon excitations. This zero point motion induces fluctuations of the initial conditions in heavy ion collisions. For example, the fluctuations associated with isovector dipole resonance account for the isobaric distributions measured in fission or fragmentation processes $17 \%$. Another example is given by subbarrier fusion which may be explained by fluctuations of the nuclear shape. 


\section{II.3. - Heavy ion dynamics.}

The existence of giant resonances which are strongly coupled to the ground state is of great importance for the description of heavy ion dynamics. For instance, Fig. 2 presents a heavy ion reaction calculated in the Copenhagen model $/ 8 /$ which explicitly takes into account giant state degrees of freedom. In these calculations the excitation of giant resonances induces deformations of the nuclei and dissipation of kinetic energy and angular momentum. Hence, giant resonances act as doorway states for thermalization.
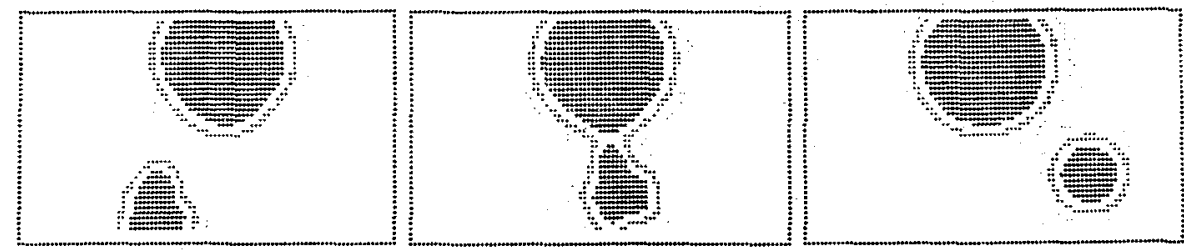

Fig. 2 - See ref. /8/

The complete understanding of the role of giant resonances in heavy ion reactions implies a more profound knowledge of their properties and excitation mechanisms.

\section{III - NEW STUDIES ON GIANT RESONANCES}

III.1. - Specificities of heavy ion probes.

In this section I shall survey the specificities, advantages and disadvantages of heavy ions in comparison with other hadronic or electromagnetic probes.

a) Dis sadvantagages

Heavy ion probes aré much more complex than light probes : the excitation mechanism is complicated and the projectile may be excited during the collision. This last point makes the ability of heavy ions to be clean probes of the nuclear structure doubtful. Indeed, a heavy ion reaction can be represented by a point in the plane defined by the two excitation energies of the target and the projectile (see Fig. 3). But in fact only the sum of these two energies is measured, which means that the inelastic spectrum is the projection of this plane on the first bisecting line. Yet, as the projectile is detected after particle decay the region above the particle threshold in the projectile must not be considered. Only the non hatched region must be projected onto the diagonal. Below Sn the projectile excitations are only Doppler broadened. Therefore the best resuits will be obtained when the projectile has a small particle threshold and no excited states under this 


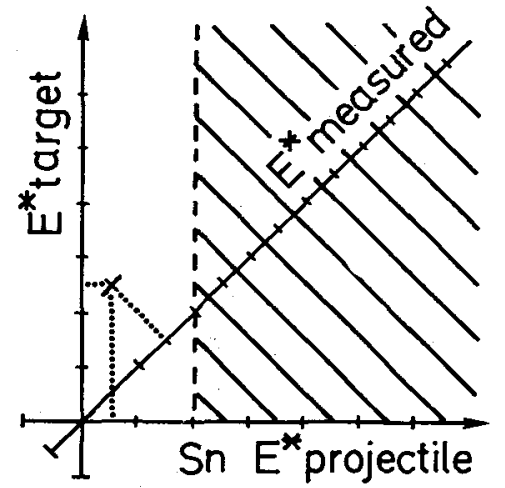

Fig. 3

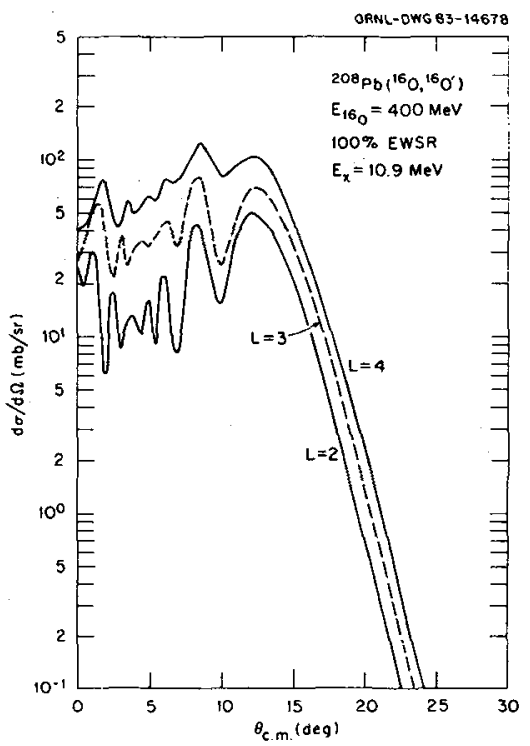

Fig. 4 - See ref. /10/

threshold (e.g. ${ }^{17} 0,{ }^{20} \mathrm{Ne}$ ). Another disadvantage of heavy ions associated with the particle decay of the ejectile is the pick-up-break-up background. In this process the projectile picks-up one or more nucleons from the target while being excited and then decays in flight be reemiting the same particles. The resulting fragment is miss detected as a scattered projectile. This process is the most important contribution to the background but can be calculated using Monte Carlo methods /9/. This background is found to be smooth in most cases and strongly dependent on the incident energy.

The last disadvantage of heavy ion probes is the absence of signature of the multipolarity of the transition. This feature is clearly illustrated in the D.W.B.A. calculation shown on Fig. 4 which shows the similarity of the angular distributions for all multipolarities in the $160+208 \mathrm{pb}$ case at $400 \mathrm{MeV}$.

\section{b) Advanantages}

Heavy ion probes also present numerous advantages for giant resonance studies. These advantages can be separated into two categories : advantages for coincidence experiments and advantages for the search of new states.

\section{Advantages for Coincidence Experiments :}

In Figure 5 which presents the inelastic spectrum of the ${ }^{20} \mathrm{Ne}+{ }^{208} \mathrm{~Pb}$ reaction at 800 MeV obtained at GANIL with the magnetic spectrometer SPEG /11/ the peak over continuum ratio of the GQR is large (at least as large as the best one obtained with other probes). This is an important point for coincidence experiments especially as the continuum is mainly the well-known pick-up-break-up background which can be eliminated in such experiments. Moreover the differential cross sections of giant states are found to be large and to grow up rapidly with the incident energy (see Fig. 6). 


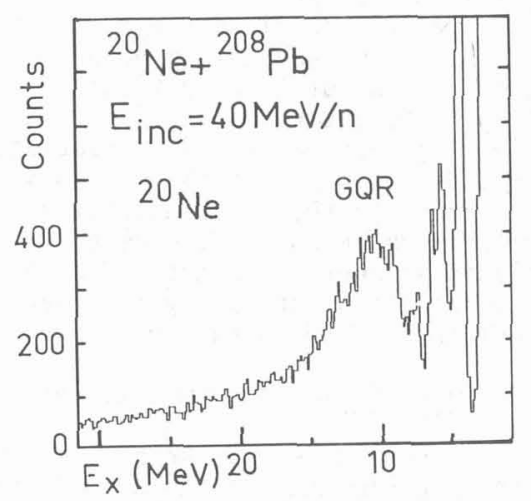

Fig. 5 - See ref. /11/

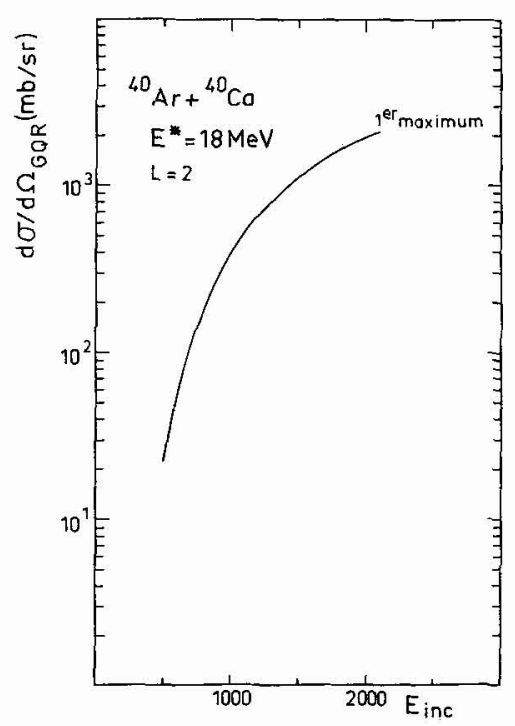

Fig. 6 - DWBA calculation for the incident energy dependence of the differential cross-section of the first maximum of angular distribution in the case of a $L=2$ state $(100 \%$ EWSR, $18 \mathrm{MeV})$ in $40 \mathrm{Ca}$ excited by an $40 \mathrm{Ar}$ projectile.

Advantages for the Search of New States: ion probes :

Several facts are in favour of the search of new giant states with heavy

i) The fieids involved in heavy ion reactions are so strong that strong excitations of the nuclei may be expected.

ii) As discussed before, the background is sma11, smooth and calculable.

iii) The large variety of projectile-ejectile couples allows to select particular spin and isospin transfers.

iv) Finally, the heavy ions bring large angular momenta into the system and thus can test high multipolarity strength distributions.

\section{III.2. - Decay studies.}

In this section I shall discuss coincidence experiments in the light of the results on $208 \mathrm{~Pb}$ described in ref. $/ 10,12 /$. Figure 7 displays the most important levels in $208 \mathrm{pb}$ and $207 \mathrm{~Pb}$ relevant for the decay studies.

\section{a) Ihe aims}

The aim of such experiments is to study the decay (i.e. branching ratios) of Giant Resonances by $\gamma$ rays and neutrons or protons. The direct measurement 


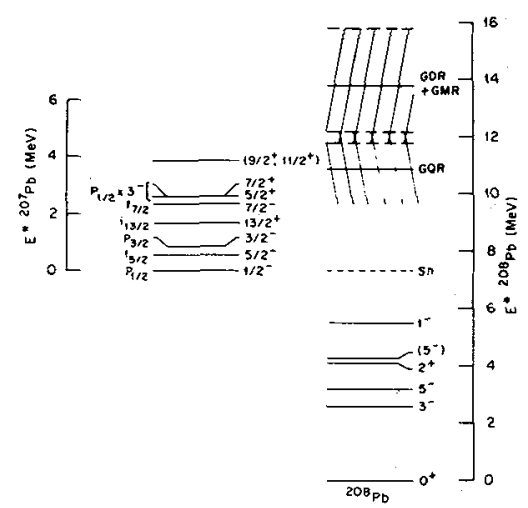

Fig. 7. - See ref. /12/

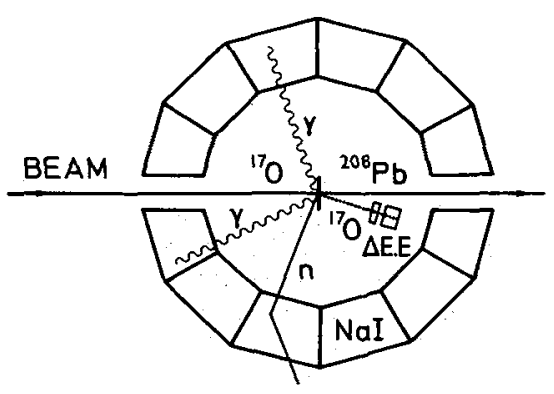

Fig. 8

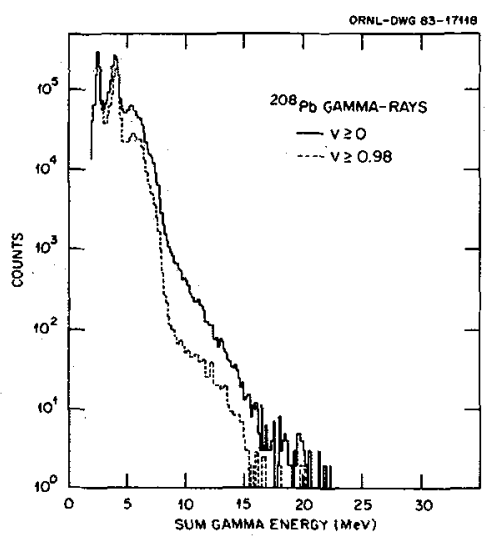

Fig. 9 - See ref. /12/ of the $\gamma$ branching ratio to the ground state gives the $B\left(E_{1}\right)$ value of the excited state. The branching ratios to excited states yield information on the underlying microscopic structure of giant states and may be helpful in the extraction of high multipolarity strength. Moreover the angutar distributions of neutrons and $\gamma^{\prime} s$ associated with a direct decay to the ground state give access to the multipolarity of the excited mode. Finally, the detailed analysis of energy spectra and of angular correlations are a way to separate direct decay from spreading decay.

\section{b) Characteristic experimenta] set up}

Figure 8 shows a schematic view of the experimental set up used in the experiment of reference $/ 12 /$. The ejectiles are detected in $8 \Delta E$. E telescopes whereas $\gamma^{\prime} s$ and neutrons are measured in a array of $\mathrm{NaI}$ detectors which covers a large solid angle $(\approx 4 \pi)$. This device separates $\gamma^{\prime}$ 's from neutrons and yields energies and multiplicities on an event by event basis. Thus the measured quantities are : i) the kinetic energy of the ejectiles (i.e. $17_{0}$ in the case of refs. $110,12 /$ equivalent to the $E^{*}$ of the target, ii) The energies of $\gamma$ 's and neutrons, $i i i)$ The $\gamma$ and neutron multiplicities. In what follows 1 shall only discuss $\gamma$ ray decay.

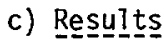

Figure 9 shows typical spectra obtained in ref. /12/. The two histograms correspond to spectra of $208 \mathrm{~Pb}$ excitations which decay by any number of $\gamma$ rays (solid line) and by only one $\gamma$ ray (dashed line). Therefore the ground state branching spectrum is obtained by taking the ratio of these two spectra (Fig. 10a). The peaks in Fig. 10a are at the positions of low Tying states in $208 \mathrm{pb}$. The bump around 10-12 MeV corresponds to the strong localization of electromagnetic strength in the giant states. This bump is a mixing of the giant quadrupole (GQR) and giant dipole (GDR) resonances and perhaps of other multipolarities. A detailed analysis of this result gives a new measurement of the $B(E 2)$ value for the $G Q R / 12 /$. 


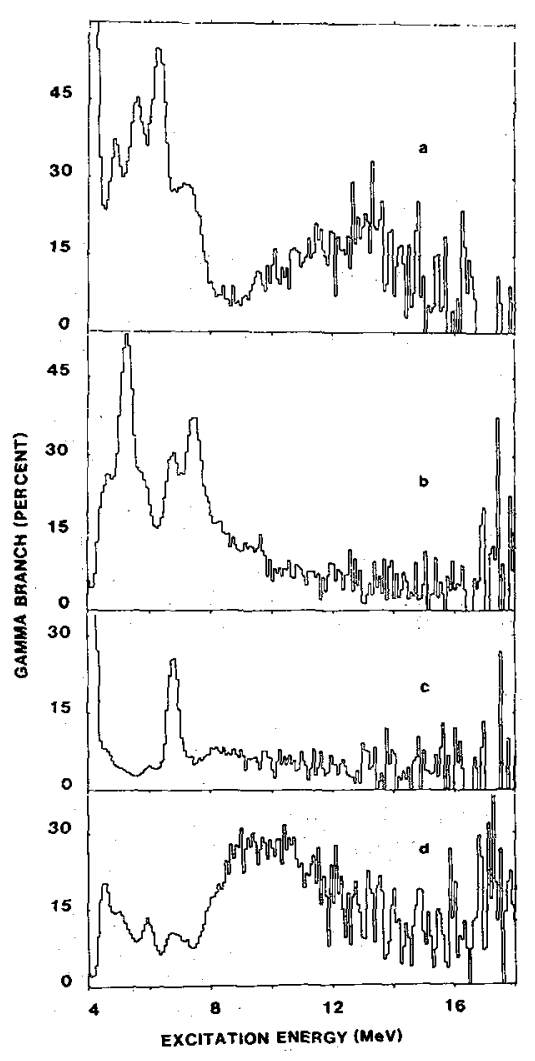

Fig. 10 - See ref. /1/

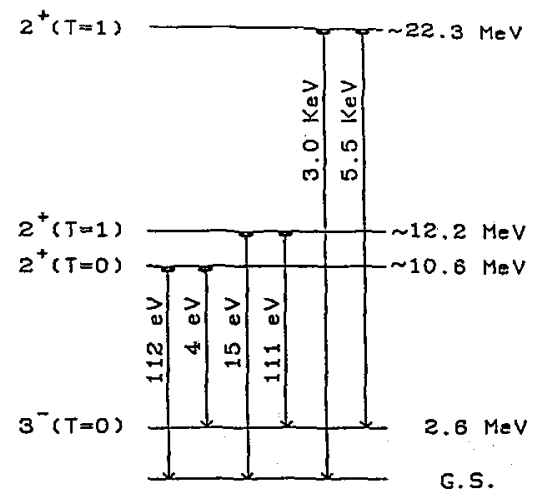

Fig, $11-\Gamma$ width for $L=2$ states in $208 \mathrm{pb}$ see ref. /14/
In fact, much more information can be extracted from such experiments. For instance, the branching ratio to all low lying states may be measured. Figure 10. gives some examples of branching ratios : i) to the ground state (part a) ; ii) to the collective $3^{-}$state at $2.61 \mathrm{MeV}$ (vart b) ; $i_{i j)}$ to the collective $2^{+}$state at $4.08 \mathrm{MeV}$ and iv) to the particle-hole $3^{-}$state at $4.97 \mathrm{MeV}$. These four spectra appear to be very different : whereas the $G Q R$ bump is clearty visible in the first one, it disappears completely in the second and third ones. These results are a strong test of. the theoretical description of giant states and in fact are in remarkable agreement with the recent calculations of Bortignon et a1. /13/. Moreover, in Fig. $10 \mathrm{~d}$, a strong decay yield is observed around $10 \mathrm{MeV}$. This feature together with the similar results obtained for the $5^{-}$ state at $3.9 \mathrm{MeV}$ seem to indicate the existence of high spin strength underlying the GQR.

In Figure 10b, it must be noted that the strong peak at 5.2 MeV might be the first experimental signature of the existence of a two phonon state (i.e. of the second excited state of a vibrational band) built on the low lying $3^{-}$state.

\section{d) Future developments}

In addition to the information on the microscopic structure of giant states coincidence experiments could be a useful tool for the search of new states such as isovector modes. Indeed it can be shown $/ 14 /$ that isovector giant quadrupole modes are strongly coupled to the ground state and to the low lying three minus state (see Fig. 11). Furthermore, in heavy ion collisions at high incident energy, these states are strongly excited by the coulomb interaction. Therefore $\gamma$-ray coincidence experiments with high energy heavy ion probes could be the best way to investigate the isovector strength. Another interesting possibility is to study charge exchange reactions because of the possible additional selection of spin transfer $/ 15 /$. 
III.3. - Search of new states.

\section{a) Theoretical_point_of view}

From a theoretical point of view numerous yet unobserved giant states are predicted (for instance : high multipolarity strength, high energy resonances, isovector modes, ...) $/ 16-19 \%$. I would like to report here the main results obtained in the multiphonon calculation of refs $/ 19,20 /$. In references $/ 19,20 /$ the response of the target nucleus to the external field created by the projectile is calculated microscopically in the quasi-boson approximation (i.e. the RPA for the first phonon). On the other hand the nuclei are assumed to follow classical trajectories. This view point is very close to the TDHF approximation /19/ or to the Copenhagen model /21/ except for the microscopic treatment of target excitations.

The calculation of a heavy ion reaction in this model must be performed in two steps. The first step consists of the microscopic description of the target response to the external field created by the projectile. For example, the continuous part of the RPA strength distribution in lead excited by an argon projectile is plotted in Fig. 12a. We have solved the RPA equation in coordinate representation with the Skyrme interaction SGII in order to obtain a good description of giant resonances together with a full treatment of continuum effects $/ 19,20 \%$. In this figure the discrete states are not displayed. The first peak corresponds to the GQR but an another bump is visible around $35 \mathrm{MeV}$. For comparison the usual distribution associated with the $r$ to the power $L$ excitation operator is displayed in Fig. 12b. In that case no high lying resonances are excited. This illustrates the fact that a reliable microscopic description of target excitations is a crucial point in such calculations.

Before concluding that new high lying resonances are excited in heavy ion reactions, one must first go throught the dynamics of these reactions. The main effect of the dynamics is to weight the strenght distribution by a kinematical cut off factor $/ 19-20 /$. The role of this factor is very important and is illustrated in Fig. 13 which gives the one phonon excitation probability for the argon on lead reaction at $11 \mathrm{MeV}$ per nucleon. As you can see, the cut off factor suppresses high energy transitions because their pulsation is missmatched with the reaction time.
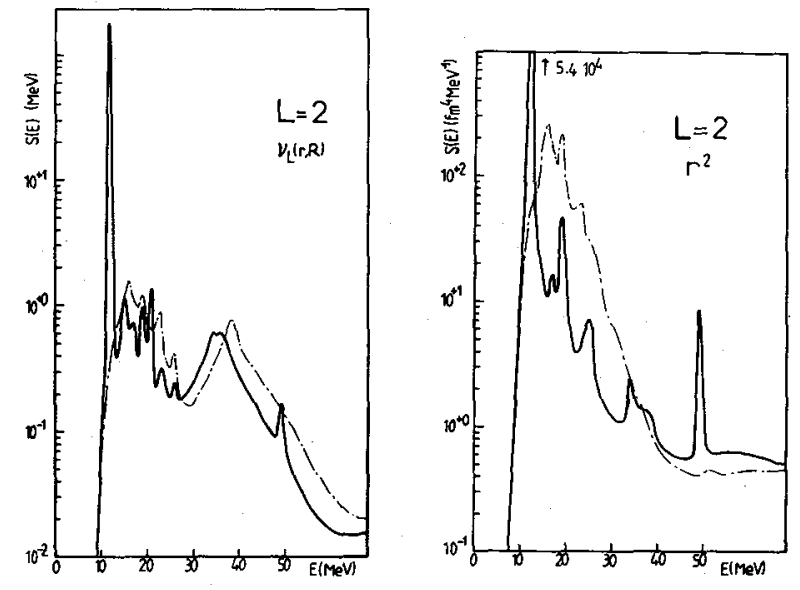

Fig. 12 - RPA strength function (solid line) and HF strength function (dotted line) for the nuclear operator $\nu_{L}$ (part a) and for the Coulomb operator $r L$ (part b) see ref. /19\%

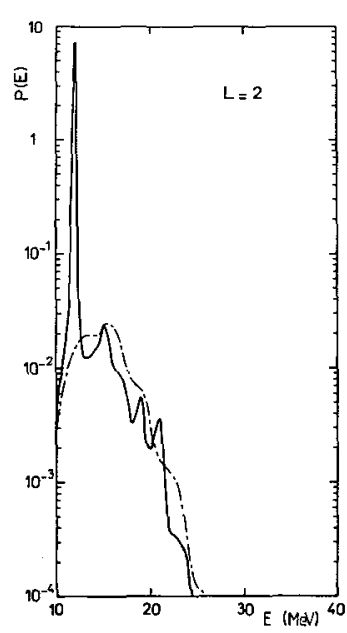

Fig. 13 - Monophonon excitation probability distribution see ref. /19/ 


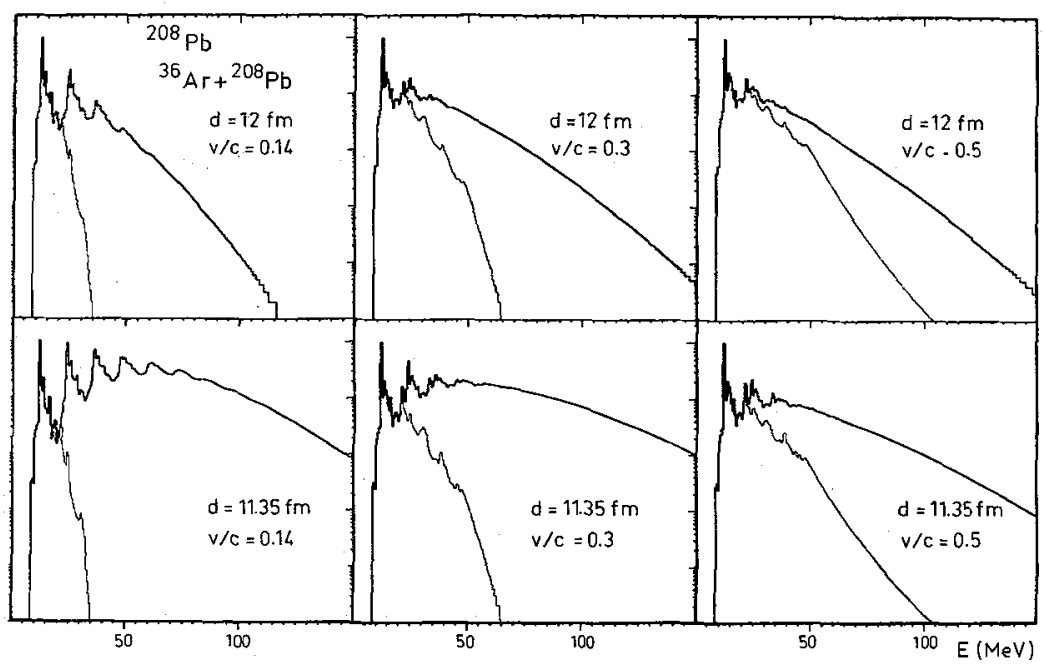

Fig. 14

The results of the multiphonon excitation calculation are summarized on Fig. 14 which shows the target excitation spectrum (thick line) of the $36 \mathrm{Ar}+208 \mathrm{~Pb}$ reaction at three incident energies and for two distances of closest approach around the grazing. The monophonon distribution (thin line) is also displayed.

At low incident energy the inelastic spectrum shows structures due to the multiple excitation of the 2 hw giant states. These multiphonon states are more strongly excited below the grazing distance of closest approach which is around $12 \mathrm{fm}$. On the other hand the monophonon distribution dominates the inelastic -spectra at high energy. Structures are also visible up to 40 or $50 \mathrm{MeV}$ but, here, they are due to high lying giant state excitation. These monophonon states are not excited at low incident energy because of the missmatch of their pulsation with the reaction time. A complete study shows that heavier projectiles favour multiphonon excitation whereas lighter projectiles strongly excite high lying monophonons /19/.

In conclusion fast light heavy ions must allow to observe the high lying giant resonances and slower heavier ions the multiphonons built with $2 \hbar \omega$ resonances.

b) Experiments with_light "heavy_ions"_at_high_incident_energy

Figure 15 shows the inelastic spectrum of the ${ }^{12} \mathrm{C}+{ }^{208} \mathrm{~Pb}$ reaction at $200 \mathrm{MeV} /$ nucleon. This reaction was performed at the Saturne facility in Saclay by Bonin et a1. At this energy giant resonances are strongly excited and the peak over continuum ratio is very high. Structures are visible up to $40 \mathrm{MeV}$ and, irrespective of some detection background $/ 24 /$, could correspond to the high energy monophonon strength extracted by the same group from $\alpha$ scattering experiments $/ 23,24 /$. 
c) Experiments with heavy "heavy ions" at moderate incident_energy

Comparison between theory and experiment at low incident energy ( $E / A \approx 10 \mathrm{MeV}$ )

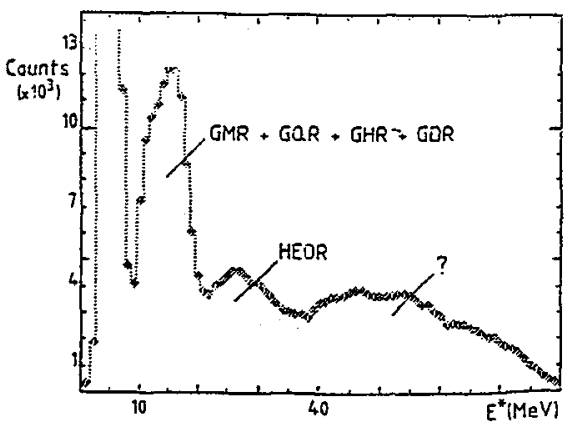

Fig. 15 - See ref. /24/

An illustration of theoretical predictions concerning the excitation of multiphonons is given by the high energy structures observed in heavy ion reactions at low energy. In references $/ 19,20 /$ it is shown that the multiphonon model provides an adequate explaination for the positions and the widths of the observed structures and the shapes and the angular evolution of the spectra. An example of this agreement is displayed on Fig. 16 which compares

- on the one hand, the total excitation probability, as in Fig. 14

- and, on the other hand, the measured spectra (this: figure displays a transfer channel which allows a clear observation of the angular evolution because of the absence of the tail of the elastic peak. Nevertheless the essential features are the same as in the inelastic channel. This means that the transfer reaction leads only to a small additional excitation on the multiphonon excitation of the core.

The remarkable feature is the striking similarity between the calculation and the data both in shape and in evolution. For impact parameters larger than the grazing only the monophonons are predicted to be excited whereas strong probabilities to excite multiphonon are found for small distances of closest approach. Likewise in the experimental spectra, only the first structures are observed at the grazing angle whereas the deeper ones become predominant at smal Ter angles.

\section{New intermediate energy results /22/}

The new results obtained at the GANIL national facility in Caen have shed light on the multiphonon excitation process. These results at intermediate energy are important because i) the variation in incident energy allows to distinguish between the pick-up-break-up process and the target excitation ii) the quasi-boson calculation predicts the optimal incident energy for the multiphonon excitation to be around 30 to $40 \mathrm{MeV} /$ nucleon.

Figure 17 presents the most complete set of data which was obtained for the $40 \mathrm{Ar}+90 \mathrm{Zr}$ reaction at $44 \mathrm{MeV} / \mathrm{nucleon}$. At angles close to the grazing bumps are clearly visible up to $60 \mathrm{MeV}$ excitation energy. The cross section decreases rapidly with angle and only a smooth background remains above $3.8^{\circ}$. The bumps appear even more clearly on the partial sums displayed on Fig. 18. In this representation at least three bumps at 50,58 and $66 \mathrm{MeV}$ are observed, the positions of which are independent of angle.

In order to get a deeper understanding of these results a Fourier analysis has been performed and is presented in Figures 19,20 . In order to eliminate the statistical fluctuations a filter is applied to the high frequencies of the Fourier transform spectrum and then the inverse Fourier transform is taken. In figure 19 the result of such an analys is is compared to the original spectrum. In the histogram which is the result of the analysis the statistical fluctuations have been eliminated and only wide bumps are observed. This analysis allows a better definition of the positions of the structures. Figure 20 presents the same analysis performed on four spectra at different angles. This figure clearly illustrates 


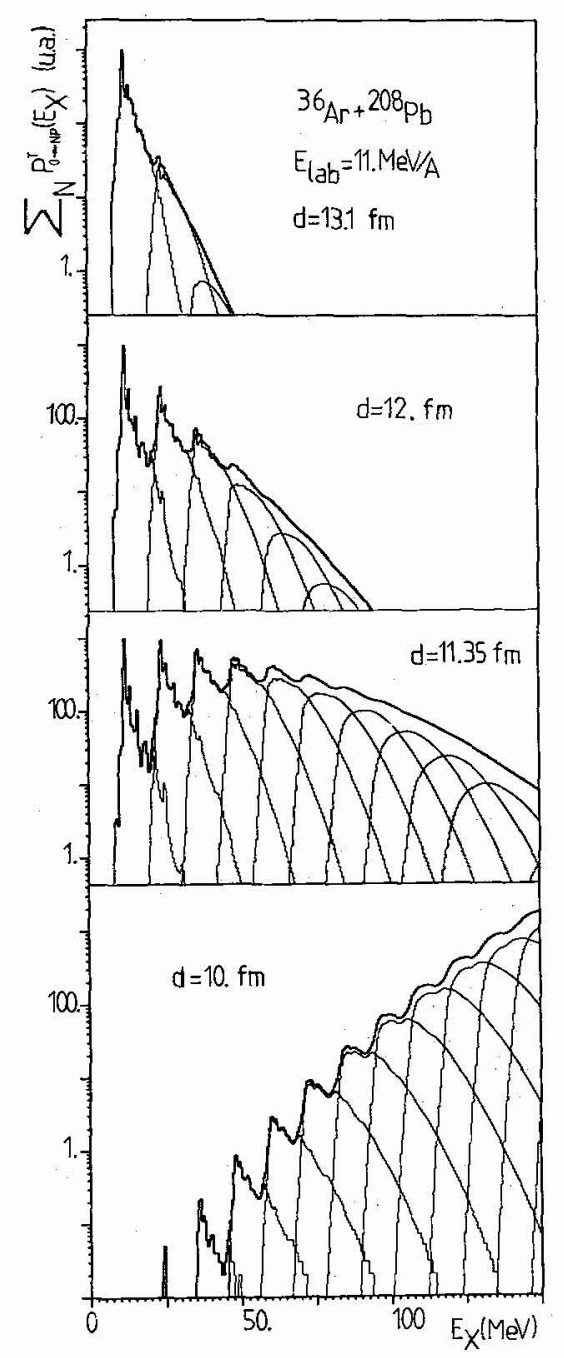

Fig. 16a

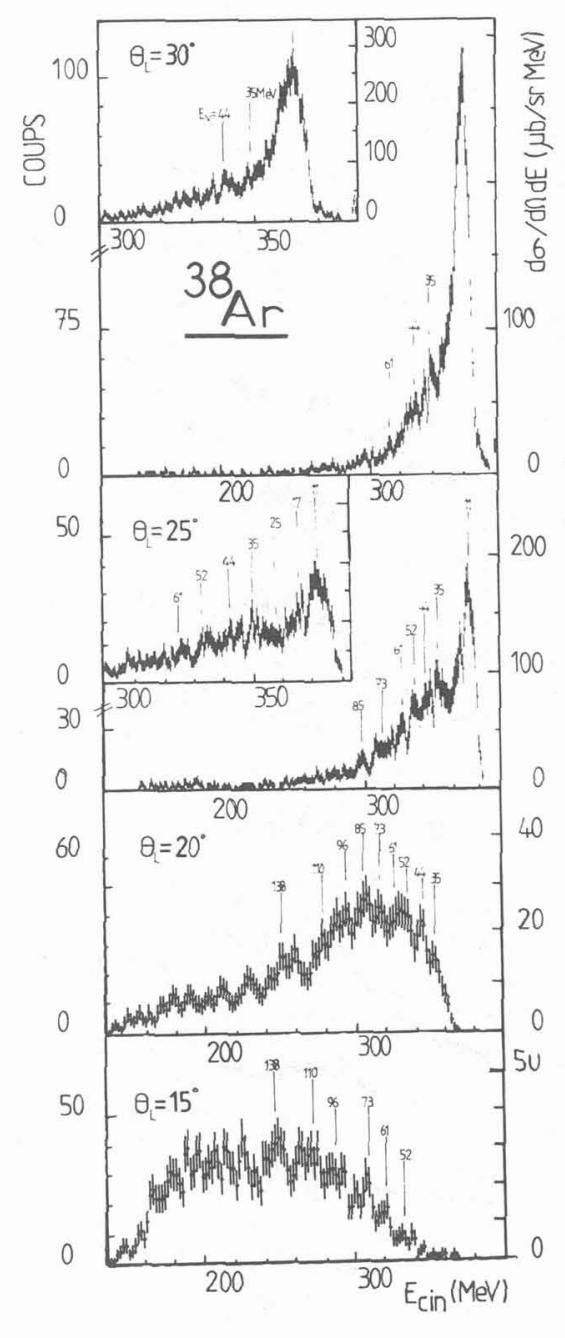

Fig. 16b

Fig. 16 - See refs $/ 19,20 /$ 


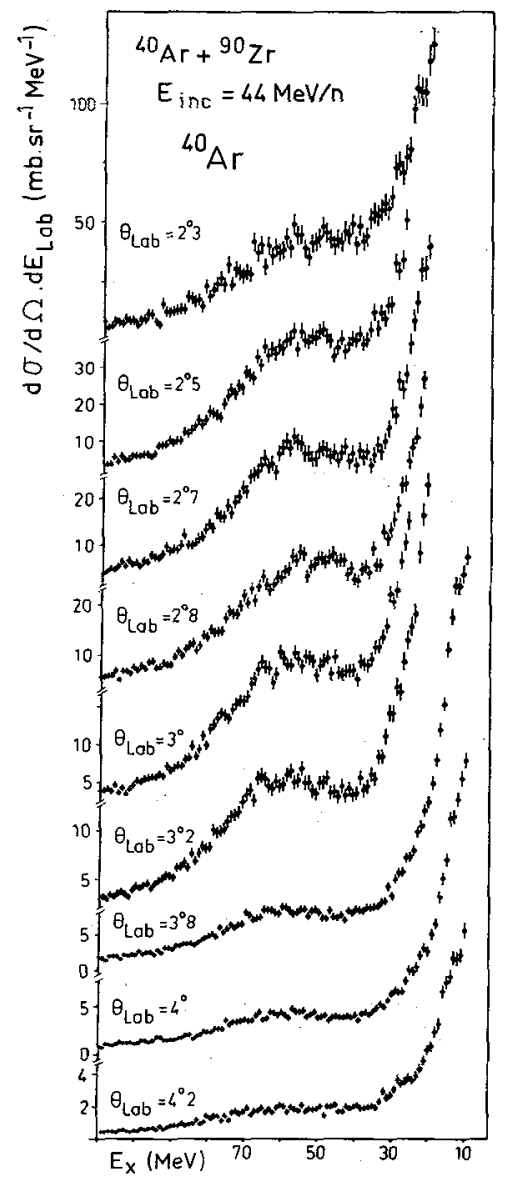

Fig. 17 - Inelastic spectra of $40 \mathrm{Ar}+{ }^{90} \mathrm{Zr}$ at $44 \mathrm{MeV} / \mathrm{n}$ see ref. $122 /$

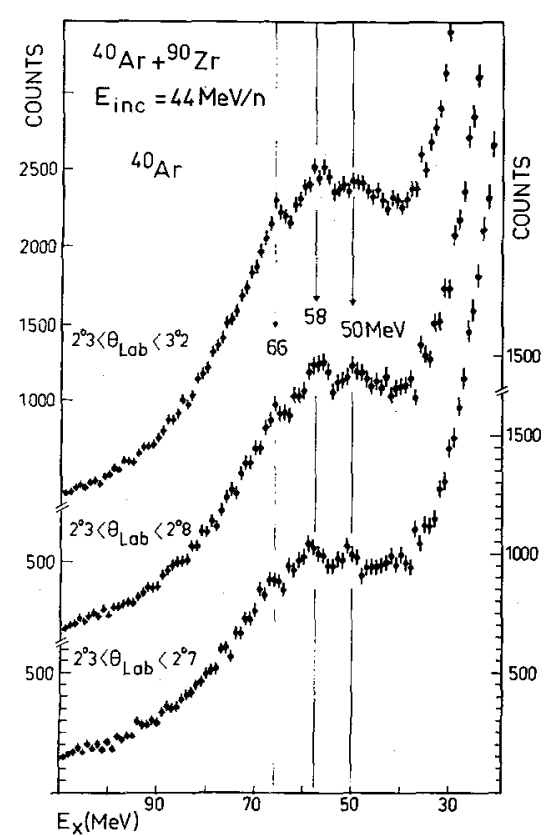

Fig. 18 


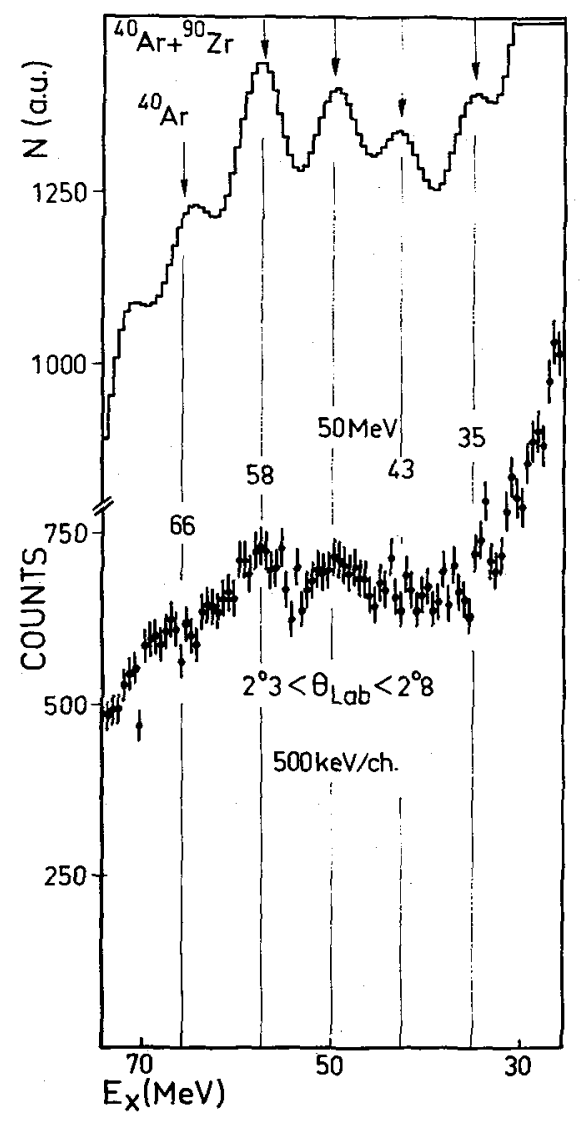

Fig. 19

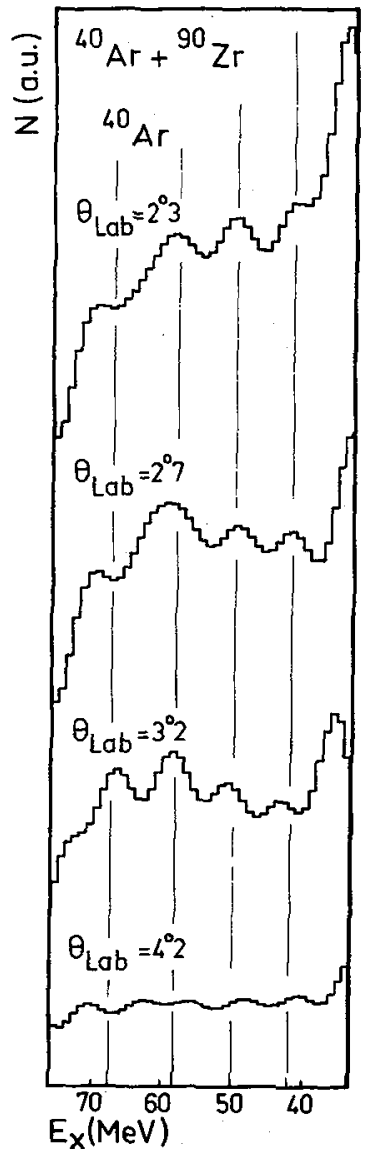

Fig. 20

the fact that the structures show up at the same excitation energy in all the spectra, except above $3.8^{\circ}$ where only a smooth background was observed in the original spectra.

\section{Influence of the incident velocity}

Another feature brought by the new results at high incident energy is given by the comparison of spectra obtained at different incident energies. For example Fig. 21 presents the inelastic channels of the $40 \mathrm{Ar}+90 \mathrm{Zr}$ reaction at 33 and $44 \mathrm{MeV} /$ nucleon. For these two inelastic spectra the pick-up-breack-up background resulting from a Monte Carlo calculation is drawn $/ 9 \%$. As expected, this background is different for the two energies. It is broadened and shifted to larger excitation energies when the incident energy is increased. Conversely the structures show up at the same positions in the two experimental spectra. Therefore the structures are independent of the incident energy. Moreover it is shown in refs. 120-22/ that they are also independent of the projectile nature. 


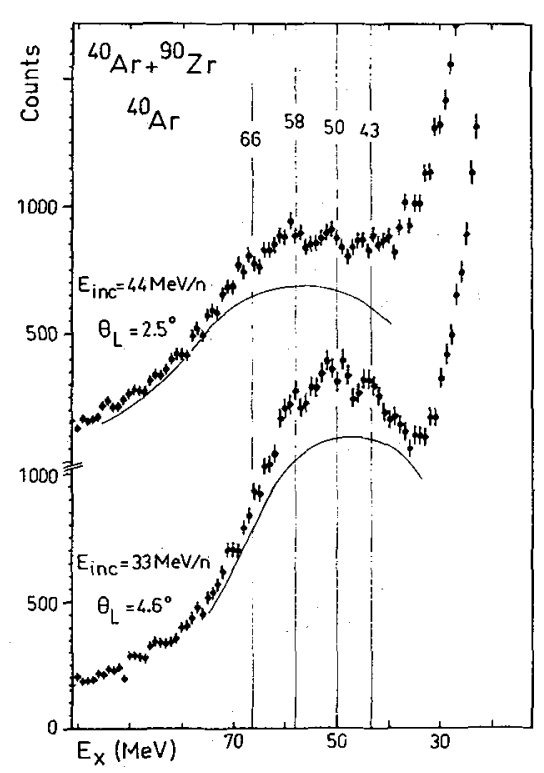

Fig. 21

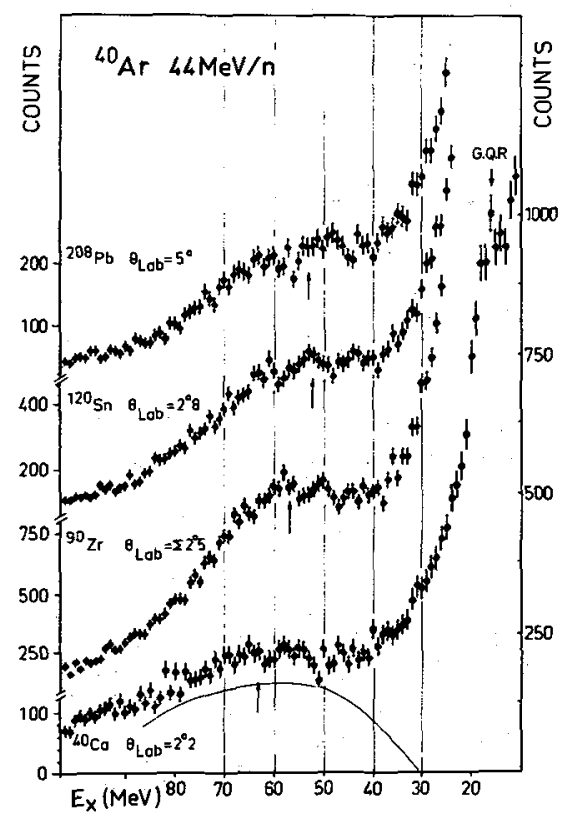

Fig. 22 - See ref. /22/
Influence of the target nucleus

In fact, the structures only depend on the target nucleus. In Fig. 22 are displayed four inelastic spectra obtained by bombarding four different targets $40 \mathrm{Ca},{ }^{9} \mathrm{Zr}$, $120 \mathrm{Sn}$ and $208 \mathrm{~Pb}$ with an $40 \mathrm{Ar}$ beam at 44 $\mathrm{MeV} / \mathrm{nucleon}$. These four spectra were measured around the respective grazing angles of the considered reaction. For the lower spectrum the pick-up-break-up background is drawn with a thin line. For the three others only the centroid of this contribution is indicated by arrows since the shapes of the four contributions are the same $19,22 /$.

In the four spectra, the structures are observed at different excitation energies. The heavier the target, the more numerous and the narrower the bumps.

This evolution is quantitatively illustrated in Fig. 23 which presents the positions of the structures as a function of the target mass $A^{-1 / 3}$. The straight lines passing through four data points and through the origin clearly show that the positions of the structures follow a $A^{-1 / 3}$ law. This is known to be a characteristic property of giant resonance states. For instance, the slopes of the three first lines correspond to the low excitation octupole resonance (LEOR), to the GQR and to the high excitation octupole resonance (HEOR) respectively. The others are not known but two of them could correspond to two already predicted /17/ and tentatively observed /24/ giant resonances at high energy. The others are in good agreement with what is expected from the multiphonon mode1 : Approximately two and three times the GQR slope for the two first Tines.

\section{Role of multiphonons in heavy ion reactions}

As we have seen, the new GANIL results have confirmed the predictions of the multiphonon model. Multiphonons seem to be strongly excited in heavy ion reactions between 10 and $50 \mathrm{MeV} /$ nucleon. This is an important feature for the heavy ion dynamics. Indeed, multiphonons appear to be the preferential way of dissipating energy and angular momentum in the first stages of the collision and in fact they can be thought of as doorway states for thermalization. Accordingly, to the extent that they induce energy dissipation and deformations in $\vec{r}$ and $\vec{p}$ spaces, multiphonons may be also important in many. other processes like fusion, fragmentation, pion production, .../25, 26/. 


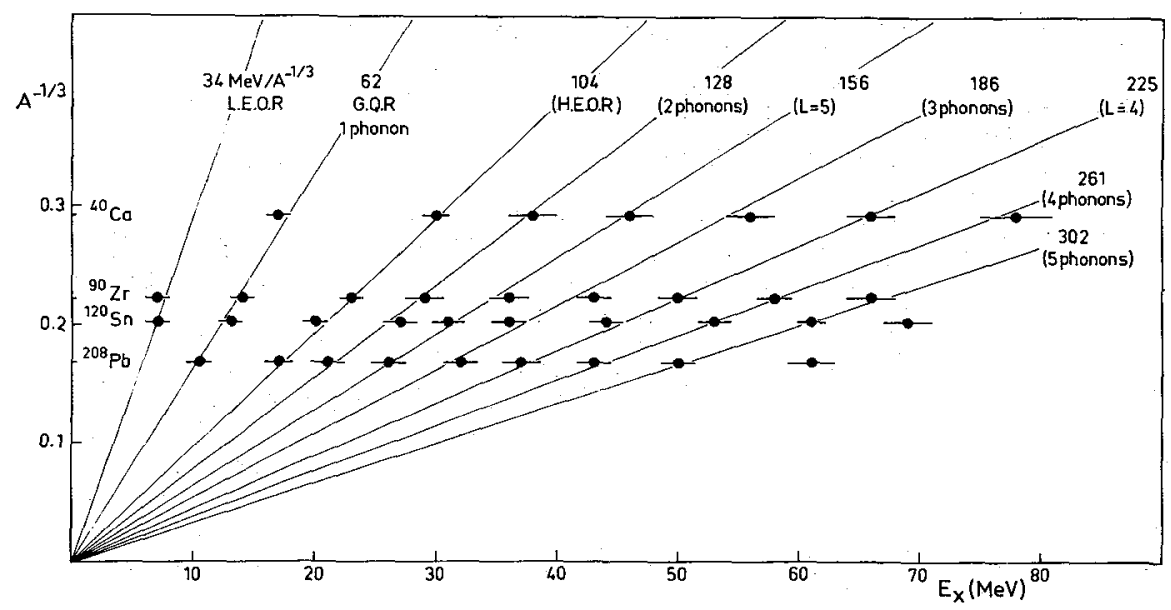

Fig. 23

IV - CONCLUSION

Irrespective of some important disadvantages heavy ions have brought and will continue to bring new information about giant resonances.

In this paper 1 have described three examples of new results, first, the study of the microscopic structure of giant states using $\gamma$ coincidence measurements, second, the search of high lying states with light "heavy ion" probes at high incident energy, and third, the investigation of multiphonon excitation using heavier ions at intermediate energy. I have al so discussed the role of giant resonances in heavy ion reactions and I have emphasized the importance of multiphonons in the dynamics of these collisions. Indeed these multiple phonon states could be the doorway towards thermalization. Moreover the deformations they induce in $\vec{r}$ and $\vec{p}$ spaces should have important consequences on the measured observables in many heavy ion reactions.

\section{Acknowledgements}

I wish to acknowledge my colleagues J. Barrette, J.C. Jacmart, B. Fernandez, J.P. Garron, J. Gastebois, W. Mittig and particularly Y. Blumenfeld, N. Frascaria, Patricia Rousse1 and J.C. Roynette for many stimulating discussions and for allowing me to use some of our unpublished results. Special thanks are due to $Y$. Blumenfeld and $N$. Frascaria for their help during the elaboration of this paper. Discussions with $H$. Flocard, Nguyen Van Giai, D. Vautherin and M. Veneroni are gratefully acknowledged.

\section{REFERENCES}

/1/ Baldwin, G.G. and Klaiber, G.S., Phys. Rev. 71 (1947) 3 and 73 (1948) 1156.

/2/ Bertrand, F.E., Nucl. Phys. A354 (1981) 129c.

13/ Buenerd, M., et al., Phys. Rev. Lett. 40 (1978) 1482.

14/ Do11, P., et a1., Phys. Rev. Lett. 42 (1979) 366.

15/ Chomaz, Ph., et al., Z. Phys. A318 (1984) 41. 
16/ Buenerd, M., La Recherche 106 (1979) 1192.

17/ Morrissey, D.J., et a1., Phys. Rev. C18 (1978) 1267.

18/ Polarolo, G., et a7., Nucl. Phys. A45T (1985) 122.

$19 /$ Biumenfeld, Y., et a1., Nucl. Phys. A445 (1985) 151.

/10/ Bertrand, F.E., J. Phys. Paris 45-C4 (1984) 99.

111/ Frascaria, N., et a1., preprint in preparation.

/12/ Beene, J.R., et al., Proceedings of "Nuclear Structure 1985", R.A. Broglia, G.B. Hagemann and B. Herskind (eds) (1985) 503.

/13/ Bortignon, P.F., Broglia, R.A. and Bertsch, G.F., Phys. Lett. 148B (1984) 20 and to be published;

Beene, J.R., et al., Phys. Lett. 164B (1985) 19.

114/ Speth, J., Cha, D., Klemt, V. and Wambach, J.," "A Clear Signature to Detect the Isovector Giant-Quadrupole Resonance", to be published.

115/ Von Oertzen, W., et al., to be published and Z. Phys. A323 (1986) 373.

116/ Liu, K.F. and Brown, G.E., NucT. Phys. A265 (1976) 385.

/17/ Dechargê, J., et a1., Phys. Rev. Lett. 49 (1982) 982.

118/ Fayans, S.A., Palichik, V.V. and Pyatov, N.I., Z. Phys. A308 (1982) 145.

119/ Chomaz, Ph., Thesis, Orsay IPNO/TH 84-01 (1984) ;

Chomaz, Ph., Nguyen Van Giai and Vautherin, D., to be published.

120/ Chomaz, Ph., et a1., Z. Phys. A319 (1984) 167.

121/ Broglia, R.A., Dasso, C.H. and Winther, A., Proceedings of the Int. School of Phys. Enrico Fermi Course LXXVII, R.A. Broglia, R.A. Ricci and C.H. Dasso (eds), North-Holland Amsterdam (1981) 327 ;

Broglia, R.A., et a1., Phys. Rep. 48C (1978) 351.

122/ Frascaria, N., Proceedings of the XXIV Int. Winter Meeting on Nuc1. Phys., Bormio (1986) to be published.

123/ Bonin, B., Thesis, Orsay (1983).

/24/ Bonin, B., et aT., preprint DPhN $n^{\circ} 2342$ (1986).

125) Borrei, $V_{\text {., et }}$ ai., to be published in $Z$. Phys.

126/ Bellini, $V_{\text {. }}$, et al., Proceedings of the XXIII Int. Winter Meeting on Nucl. Phys., Bormio (1985). 